\title{
A Simple Metric of IQA with Contourlet transformation
}

\author{
Shuang Liang ${ }^{1,}$, Guanxiang Wang ${ }^{1, b}$ \\ ${ }^{1}$ School of Mathematical Science, Peking University \\ a liangshuang12@pku.edu.cn, ${ }^{\mathrm{b}}$ gxwang@math.pku.edu.cn
}

Keywords: Image quality assessment; Contourlet transformation; tid2013

\begin{abstract}
Although there are already a number of metrics of image quality assessment (IQA), most of them are complex and time consuming. This article proposed a simple method to qualify images' quality with full references. The proposed metric is tested on image database tid2013.
\end{abstract}

\section{Introduction}

Image quality assessment (IQA)is an important aspect of image application and processing. Since subjective IQA takes unaffordable time and cost, objective IQA in necessary. Usually, objective IQAs are called full-reference (FR), reduced-reference (RR) and no-reference (NR) metrics [1] based on the amount of reference information available. This article focuses on full-reference IQA.

A number of FR IQA metrics has been constructed. Of them, the conventionally and extensively used ones are FSIM [2], MSSIM [3], NQM [4], structural similarity index measurement (SSIM) [5],VIFP [6],VSNR [7],WSNR[8], peak signal-to-noise ratio (PSNR) [9], PSNR-HVS [10], PSNR-HVS-M [1q], PSNR-HA and PSNR-HMA [12]. Other more metrics have been introduced in [13].

FSIM is a component-wise metric. SSIM, structural similarity index measurement, has received extensive attention. It separates the image quality assessment into three components: luminance, contrast and structure [6], which are combined into the overall index by dot product. Then the corresponding exponents are parameters indicating the relative importance of the three parts. Finally the SSIM index map is pooled into a quality score. Now that SSIM has attracted increasing attention, some substantial attempts of improvement appear. For example, multi-scale structural similarity index (MSSIM) is proposed in [4].The authors decompose the image into multi-scale and calculate the contrast and structural comparison at each scale except that the luminance comparison is computed only at the highest scale. Human visual system (HVS) has also been employed in IQA. Though the understanding and exploration of HVS is not complete and clear, there are some HVS-based IQA metrics with good performance. For example, Chandler and Hemami proposed visual signal-to-noise ratio (VSNR) in [8] where near-threshold and supra-threshold properties of HVS are taken to quantify the visual fidelity.

Here, this article presents a full reference (FR) IQA metric simply by the difference between the coefficient matrices of contourlet transform of the reference and the distorted images.

\section{A Simple Metric of IQA with Contourlet transformation}

Contourlet transformation has been introduced to IQA for a while in all of FR, RR and NR. Wen $\mathrm{Lu}$ et al. first employed contourlet in RR image quality assessment in [14], where contourlet is combined with contrast sensitivity function (CSF) and just noticeable difference (JND) threshold to get the visual sensitivity coefficients in each subbands. And later, they proposed an image quality factor with multiscale geometric analysis [15]. They also utilized contourlet transformation in NR image quality assessment [16]. Representing the relationship of contourlet coefficients by joint distribution and using image-dependent threshold, they calculate the final IQA score with the nonlinear combination of the extracted features. Xu Wang et al. also applied contourlet transformation to RR image quality assessment [17], where a hidden Markov model-Gaussian scale mixtures (GSM) is combined with CT. Another NR IQA metric is proposed by Bin Wang [18]. He 
modeled the contourlet coefficients by generalized Gamma distribution (GGD). Then the final score is gotten with a support vector machine fed with a feature vector resulted from GGD. The so called multiscale directional difference (MSDD) metric of FR IQA based on contourlet coefficients was proposed by Mingna Liu and Xin Yang [19]. Their IQA score is obtained by pooling the masking normalized error between the contourlet coefficients of the reference and distorted images, where the used mask is Daly Semi local Mask. Since SSIM is very popular in FR IQA, Zhidan Yan et al. [20]and Sun Han et al. [21] proposed the contourlet based SSIM respectively. Both of them calculate SSIM in the subbands of contourlet coefficients, but with different weighted sums. In addition, Pan Wang et al. proposed a FR IQA metric based contourlet and saliency map[22].

For simplicity, our operation is taken only on gray images or on the luminance component of color images.

\section{The Proposed Method}

Let $I_{r}$ and $I_{d}$ denote the reference image and the distorted image respectively. The 3-level contourlet decomposition of $I_{r}$ and $I_{d}$ are denoted by $R C_{1}^{j}, R C_{2}^{j}, R C_{3}^{j}, D C_{1}^{j}, D C_{2}^{j}, D C_{3}^{j}, R C_{0}$ and $D C_{0}$, where $j=1,2, \ldots, 8 . R C_{i}^{j}$ and $D C_{i}^{j}$ represent the contourlet coefficient matrices of the $j$ th direction at $i$ th level decomposed from $I_{r}$ and $I_{d}$ respectively, and $R C_{0}$ and $D C_{0}$ are the low frequency components of the decompositions. The difference between $I_{r}$ and $I_{d}$ includes two parts, difference of high frequency and difference of low frequency. As contourlet decomposition displays the difference in multi-direction and multi-scale, we think the most difference among the directions and the scales play the significant role in qualification of the distorted image. So let

$$
H_{\text {dif }}=\max _{1 \leq i \leq 3,1 \leq j \leq 8}\left\{\left|R C_{i}^{j}-D C_{i}^{j}\right|\right\}
$$

Of course, the difference of the low frequency component, $L_{d i f}=\left|R C_{0}-D C_{0}\right|$, is important too. The quality score of the distorted image is obviously reciprocal to these two differences, but which one contributes more to the quality score is should tested. Therefore, we set the final metric of the distorted image as

$$
S=\exp \left(-H_{\text {dif }}\right)+\beta \exp \left(-L_{\text {dif }}\right)
$$

where $\beta$ is the weighting factor and it is chosen to be 0.022 by experiment in this article. It is should be noted that the Orders of magnitude of $H_{d i f}$ and $L_{d i f}$ are not same, which is one of the reasons that $\beta$ is small.

The usual way to quantify an IQA metric performance is to calculate the correlation coefficient between the predicted scores from the metric and the subjective scores. The performance criteria recommended by Video Quality Experts Group (VQEG) are prediction accuracy, prediction monotonicity and prediction consistency. And these criteria become the customary performance criteria of image quality assessment now. The prediction accuracy measures the correlation between the predicted scores and subjective scores. For which, the Pearson correlation coefficient (PCC), root mean square error (RMSE)and mean absolute error (MAE) are recommended. A nonlinear transformation is usually applied before the calculation of prediction accuracy to ensure that the predicted and subjective scores are on the same scale. The prediction monotonicity specifies the similarity on the rank-ordering of the objective and subjective scores. For which, the Spearman rank-order correlation coefficient (SROCC) and the Kendall rank-ordering correlation coefficient (KROCC) are recommended by the image database TID2013. Among the existing image databases for IQA, tid2013 is considerably suitable for testing a metric because it concludes sufficient images and sufficient types of distortion. And the subjective score provided in TID 2013 is the mean opinion score (MOS). 
Table 1. Comparison of performances of the proposed metric to some well known metrics

\begin{tabular}{ccc||ccc}
\hline Metric & SROCC & KROCC & Metric & SROCC & KROCC \\
\hline WSNR & 0.5796 & 0.4463 & VSNR & 0.6809 & 0.5077 \\
VIFP & 0.6084 & 0.4567 & MSSIM & 0.7872 & 0.6079 \\
PSNR-HVS-M & 0.6246 & 0.4818 & PSNRc & 0.6869 & 0.4958 \\
NQM & 0.6349 & 0.4662 & FSIM & 0.8007 & 0.63 \\
SSIM & 0.637 & 0.4636 & PSVR-HMA & 0.8128 & 0.6316 \\
PSNR & 0.6395 & 0.47 & PSNR-HA & 0.8187 & 0.6433 \\
PSNR-HVS & 0.6536 & 0.5077 & proposed & $\mathbf{0 . 7 3 8 1}$ & $\mathbf{0 . 5 5 9 4}$ \\
\hline
\end{tabular}

Besides our result, the data in table 1 are all from [13]. Table 1 shows that, our proposed metric performs better than most of the well known metrics though it is not the best. Taking the simplicity into consideration, the proposed metric is promising.

In some cases, another correlation coefficient, Pearson correlation coefficient (PCC) is used to qualify a IQA metric. But it is difficult to compare two metrics with PCC because a nonlinear regression is needed to compute this correlation coefficient. For the sake of fairness, we compare here the PCCs of metrics without nonlinear regression. Fortunately, the computing codes of quality scores of some metrics have been published in [23]. We list in table 2 the PCC of our metric with those of some other metrics gotten by the public codes from [23].

Table 2. Comparison of PCC

\begin{tabular}{cccccccccc}
\hline Metric & MSSIM & NQM & PSNR & SNR & SSIM & VIF & VIFP & WSNR & Proposed \\
\hline PCC & 0.7768 & 0.6275 & 0.4504 & 0.4401 & 0.6527 & 0.7337 & 0.6074 & 0.512 & 0.7289 \\
\hline
\end{tabular}

As the above table, it can be seen that our proposed metric performs in PCC better than other ones except MSSIM.

\section{Summary}

In summary, this article presents a very simple way of qualifying an image. And its performance on TID2013 shows its potential in IQA.

\section{References}

[1] Zhou Wang, Alan C.Bovik, Modern Image Quality Assessment, Morgan \& Claypool Publishers, 2006.

[2] L. Zhang, L. Zhang, X. Mou, and D. Zhang, FSIM: a feature similarity index for image quality assessment, IEEE Transactions on Image Processing, Vol. 20, No 5, pp. 2378-2386, 2011, http:// www4. comp.polyu.edu.hk/ cslzhang/IQA/FSIM/FSIM.htm

[3] Z. Wang, E. P. Simoncelli, and A. C. Bovik, Multi-scale structural similarity for image quality assessment, in Proc. IEEEA silomar Conf. Signals, Syst. Comput., pp. 1398-1402, 2003.

[4] N. Damera-Venkata, T. Kite, W. Geisler, B. Evans, andA. Bovik Image Quality Assessment Based on a Degradation Model, IEEE Transanction on Image Processing, Vol. 9, pp. 636-650, 2000. [5] Z. Wang, A. Bovik, H. Sheikh and E. Simoncelli, Image quality assessment: from error visibility to structural similarity, IEEE Transactions on Image Processing, Vol. 13, Issue 4, pp. 600-612, 2004. 
[6] H. R. Sheikh and A. C. Bovik, Image Information and Visual Quality, IEEE Transactions on Image Processing, Vol. 15,pp. 430- 444, 2006.

[7] D.M. Chandler, S.S. Hemami, VSNR: A Wavelet-Based Visual Signal-to-Noise Ratio for Natural Images, IEEE Transactionson Image Processing, Vol. 16 (9), pp. 2284-2298, 2007.

[8] T. Mitsa, K. Varkur, Evaluation of contrast sensitivity functions for the formulation of quality measures incorporated in half toning algorithms, IEEE International Conference on Acoustic, Speech, and Signal processing, Vol. 5, pp. 301- 304, 1993.

[9] Zhou Wang, Alan C.Bovik, Modern Image Quality Assessment, Morgan \& Claypool Publishers, 2006.

[10] K. Egiazarian, J. Astola, N. Ponomarenko, V. Lukin, F. Battisti,and M. Carli, New full-reference quality metrics based onHVS, Proc. of the Second International Workshop on Video Processing and Quality Metrics, Scottsdale, 4 p, 2006.

[11] N. Ponomarenko, F. Silvestri, K. Egiazarian, Carli M., AstolaJ., Lukin V., On between coefficient contrast masking of DCT basis functions, Proc. of the Third International Workshop on Video Processing and Quality Metrics, USA, 2007, 4 p.

[12] N. Ponomarenko, O. Eremeev, V. Lukin, K. Egiazarian and M. Carli, Modified image visual quality metrics for contrast change and mean shift accounting, Proceedings of CADSM, Ukraine, pp.305-311, 2011.

[13] N. Ponomarenko, O. Ieremeiev, V. Lukin, K. Egiazarian, L. Jin, J. Astola, B. Vozel, K. Chehdi, M. Carli, F. Battisti, C. C. J. Kuo, Color image database TID2013: peculiarities and preliminary results, in: Fourth European Workshop on Visual Information Processing (EUVIP), 2013, pp.106-111.

[14] Wen Lu, Xinbo Gao, Xuelong Li, Dacheng Tao, An Image Quality Metric Based Contourlet, 15th IEEE International Conference on Image Processing, 2008.

[15] Xinbo Gao, Wen Lu, Dacheng Tao, Xuelong Li, Image Quality Assessment Based on Multiscale Geometric Analysis, IEEE Transactions on Image Processing, Vol.18, No.7, 2009.

[16]Wen Lu, Kai Zeng , Dacheng Tao, Yuan Yuan, Xinbo Gao, No-reference Image Quality Assessment in Contourlet Domain, Neurocomputing, Vol.73, Issues 4-6, Jan 2010, pp.784-794.

[17]Xu Wang, Gangyi Jiang, Mei Yu, Reduced Reference Image Quality Assessment Based on Contourlet Domain and Natural Image Statistics, 5th International Conference on Image and Graphics, 2009.

[18]Bin Wang, Image Quality Assessment Based on Contourlet Transform and Generalized Gamma Distribution, 3rd International Conference on Material, Mechanical and Manufacturing Engineering (IC3ME 2015).

[19] Mingna Liu, Xin Yang, Image Quality Assessment Using Contourlet Transform, Optical Engineering, 48(10), October 2009.

[20]Zhidan Yan, Junxiang Li, Chunmei Yang, The Contourlet-based Structural Similarity for Image Quality Evaluation, 5th International Conference on BioMedical Engineering and Informatics, 2012. [21]Sun Han, Liu Yanrui, A New Image Quality Assessment Algorithm Based on Contourlet Transformation, 2013 Second International Conference on Robot, Vision and Signal Processing [22] Pan Wang, Wei Wu, Xiaomin Yang, Kai Liu and Gwanggil Jeon, Image Quality Assessment with Saliency Map in Nonsubsampled Contourlet Transform Domain, International Journal of Multimedia and Ubiquitous Engineering, Vol.11, No.6(2016),pp.349-360.

[23] M. Gaubatz, Metrix MUX Visual Quality Assessment Package, in 〈http://foulard.ece.cornell. edu/gaubatz/metrix_mux//, 27 March 2014. 